$$
\cos 1=.53102 .29--4
$$

UCRL- SOIOS

DES4 004157

RECONNECTION IN SPHEROMAK FORHATION AND SUSTAINMENT

James H. Hammer

This paper was prepared for submittal to the Proceedings for the AGU Chapman Conference on Magnetic Reconnection, Los Alamos National Laboratory, Los Alamos, NM, October 3-7, 1983

December 12, 1983

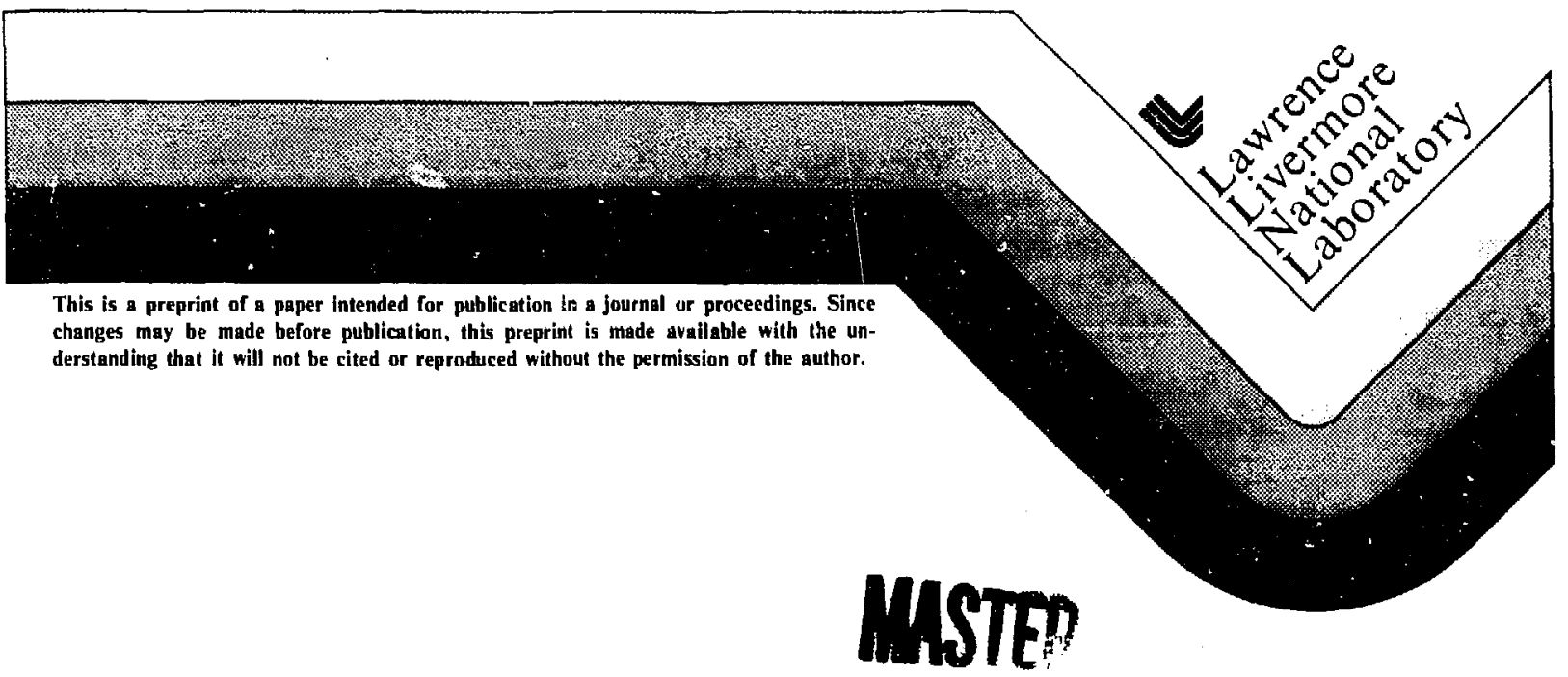




\title{
RECCNNECTION IN SPHEROMAK FORMATION AND SUSTAINMENT
}

\author{
James $\mathrm{H}$. Hammer
}

\author{
Lawrence Livermore National Laboratory \\ Livermore, $C A$
}

\section{DISCLAIMER}

This report was prepared as an account of work sponsored by an agency of the United States Government. Neither the United States Government nor any agency thereof, nor any of their employees, makes any warranty, express or implitd. or ussumes any legal liability or responsibility for the accuracy, completeness, or usefulness of any information, apparatus, product, or process disclosed, or represents that its use would not infringe privately owned rights. Reference herein to any specific commercial product, process, cr service hy trade name, trademark. manufacturer, or otherwise does not necessarily constitute or imply its endorsement, recommendution, or favoring by the United States Government or any agency thereof. The views and opinions of authors expressed herein do not necessarily state or reflect those of the United States Government of any agency thcreof.

\section{NOTHCE \\ PORTIOHS OF FHIS REPOAT ARE ILLEGIBLE. \\ It has been reproduced from the best avallable copy to permit the broadest possible availability.}


Introduction

The Spheromak is a magnetic confinement device that is being explored in both the U.S. and Japanese fusion programs. It is a member of the "Compact Torus" family of magnetic structures characterized by a set of closed, nested toroidal flux surfares but withuut any coils, transformer cores, etc. protruding through the hole in the torus. The Spheromak is closely related to the Reversed Field Pinch (RFP) in that most of the magnet ic field is produced by plasma currents flowing along the magnetic: field lines (a near force free field) rather than by external coils. The Spheromak has magnetic field comfonents of comparable strength in both the toroidal (azimuthal) and poloidal (in the plane perpendicular to the azimuthal unit vector) directions. The large internal magnetic energy in the Spheromak makss it rich in magnetohydrodynamic phenomena and reconnection, in particular, plays an important role in the formation, resistive decay and instability processes.

\section{Formation}

Spheromaks have been formed successfully in a surprisingly large number of ways. At least three completely distinct methods have worked: inductive formation using a flux core [1]; combination $Z$ and theta pinch formation [2]; and magnetized plasma gun formation $[3,4,5]$. Each of these methods, illustrated in Figs. 1, 2, 3, involves reconnection in some fashion. In the pre-formation state poloidal field lines are wrapped around or penetrating solid bodies. During formation, plasma is created and the toroidal component of the field is introduced. Magnetic forces cause the flux surfaces to distort and eventually tear off to form the desired set of closed, nested surfaces.

Magnetic probe data has confirmed the existence of closed surfaces and hence demonstrates that some form of reconnection has occurred. Fig. 4 shows 
a poloidal flux surface map from a 2 -theta pinch experiment following formation [2] and Fig. 5 shows the evolution of contours of toroidal magnetic field $\left(B_{T} \times\right.$ radius is approximately a function of pcloidal flux alone for this slow formation technique) for a flux core experiment [1]. In Fig. 6, the axial magnetic field, $B_{z}$, is shown as a function of axial position, $z$, along the axis of symmetry at various times for a plasma gun experiment [4]. The field in the entrance region between the gun muzzle and the flux conserving container decays rapidly (Fig. óa), corresponding to reconnection of the field as depicted in Fig. 3. The field in the Spheromak following reconnection (Fig. 6b) decays on a much slower time scale. In one gun experiment [5] the reconnection of field lines in the entrance region was forced to occur on an even more rapid time scale by a fast, single-turn pinching coil wrapped around the entrance region. Fig. 7a shows the time behavior of the magnetic field due to the pinching coil at a point on the symmetry axis in the middle of the entrance region, with the plasma gun turned off. Fig. $7 b$ shows the field due to the plasma gun alone and $\mathrm{Fig}$. 7c shows the combined field with both the plasma gun and pinching coil. The reconnection with the pinching coil on occurs at a rate 6 times faster than the unforced rate, i.e., in about $10 \mu \mathrm{sec}$, which is still many times the radial Alfuén transit time.

In the "classical" view of these formation methods ("classical" refers to the dominant view held several years ago) the reconnection is axisymmetric and two-dimensional in nature. A consequence of this view is that the poloidal magnetic flux contained in the resulting Spheromak should be less than or equal to the poi, idal flux linking the formation device. In many instances, however, the poloidal flux measured in the Spheromak does not conform to the classical prediction and may be as much as ten times the initial poloidal flux [5]. This phenomenon, known as "flux amplification" implies that three-dimensional 
effects are playing a big role: toroidal flux is being twisted into poloidal flux, breaking symmetry during formation.

Flux amplification can be understood quantitatively by applying Taylor's relaxation theory [6], originally developed for the RFP. The theory maintains that during the MHO turbulent formation process, many of the flux invariants of ideal MHD are broken by reconnection, leaving only the global magnetic helicity, $K=\int \underline{A} \cdot \underline{B} d V$, invariant. The magnetic energy of the systen $W=j B^{2} / 2 d V$ is then minimized subject to the constraint that $k$ alone is conserved. The helicity is unambiguously defined if the gauge is restricted to cases where $\& \underline{A} \cdot \underline{d l}=$ flux enclosed by the curve, and all flux lines are restrained to the plasma volume, i.e., no flux penetrates bounding surfaces. To understand the special significance of the helicity, it is worthwhile to briefly review its relationship to the topological linkage of the magnetic field.

Consider an infinitesimal flux tube with a half twist in it as shown in Fig. 8a. It's assumed, for simplicity, that there is no internal twist to the tube, i.e., the lines do not spiral about the center of the tube. We can evaluate $k$ for the tube,

$$
K=\int \underline{A} \cdot \underline{B} d V=\oint \underline{A} \cdot \underline{d l} B d A=d \psi \oint \underline{A} \cdot \underline{d l}
$$

where $d \psi$ is the flux within the tube and the integration path is shown in Fig. 8b. The integral can be evaluated by adding and substracting pieces to the curve as shown in Fig. $8 \mathrm{c}$. The first of the curves in Fig. $8 \mathrm{c}$ gives no contribution to the integral since it encloses no magnetic flux. This can be seen by breaking the curve up into a number of closed loops between the reference plane and the curve. The second curve in Fig. 8 c encloses the flux tube and hence gives $S \underline{A} \cdot \underline{d l}=d \psi$ and so the helicity is

$$
k=d \psi^{2}
$$




\section{$-5-$}

Similar arguments for two tubes of flux $d \psi_{1}, d \psi_{2}$ allow evaluation of the helicity. If the tubes are linked $k=2 \mathrm{~d} \psi_{1} \mathrm{~d} \psi_{2}$, whereas if they are unlinked $k=0$. More complex structures such as knots, etc., can be evaluated by simply projecting the three dimensional curves onto a plane and examining the crossings. The helicity thus corresponds to a net twist, linkage or "knotted-ness" of magnetic flux.

If infinitely conducting plasma fills the tubes, then the dyna,rical equations predict that the helicity of each tube separately will remain constant in time. A small amount of resistivity, however, will allow reccnnection of tubes with each other, without destroying global properties. Fig. 9 shows how recunnection can transform one type of helicity, in this case a simple knot, into another, a series of loops. Note that while the topology alters, the total value of the helicity is preserved. If many tubes are present, knots and twists, etc., can move from one tube to another by reconnection and local conservation of the helicity on each tube will be destroyed. In the case of complete intermixing and reconnection, only the global helicity, the helicity summed over all the tubes is preserved. The generalization of $k$ to the case of arbitrary gauge and to flux penetrating bounding surfaces is discussed in the appendix.

Fig. 10 illustrates schematically how these ideas apply to flux amplification in the plasma gun formation of a Spheromak. The plasma column can deform in a kink-like motion and twist toroidal flux into the poloidal plane. Reconnection then allows for relaxation to an axially symmetric state.

The relaxed state in the Taylor theory is described as having minimum magnetic energy for the given total helicity. An immediate consequence is that $\nabla \times \underline{B}=\mu \underline{B}$ 
where $\mu$ is a constant in space. In Fig. 11, the solutions to this equation (solid lines) are compared to the observed field profiles [5] showing good agreement. Figure 12 shows the predicted values of poloidal flux in the Spheromak for a given input of helicity

$$
k_{\text {in }}=2\left(\int v d t\right) \psi_{g}
$$

where $V$ is the voltage across the electrodes and $\psi_{g}$ is the poloidal flux threading the gun. Quantities linear in the field such as the poloidal flux scale roughly as the square root of $k$, so $\psi_{p}$ is plotted versus $(s \mathrm{vdt})^{1 / 2}$ with $\psi_{9}^{1 / 2}$ held fixed in Fig. 12 a and versus $\psi_{g}^{1 / 2}$ with $(s \vee d t)^{1 / 2}$ held fixed in Fig. 12b. The solid and dashed theoretical curves correspond to different allowances for ohmic loss and a low energy cutoff when the capacitor bank energy is insufficient to stretch the gun flux into the flux conserver.

\section{Resistive Decay and Sustainment}

During the resistive decay of the Spheromak, the profiles can drift away from the preferred (minimum energy) state. Figure 13 shows the toroidal and poloidal field versus time during the decay phase of a gun produced Spheromak [4]. At about $130 \mu \mathrm{sec}$, a fairly abrupt change occurs, bringing the ratio of toroidal to poloidal fields back to the ratio in the minimum energy state - (the dashed line). The circles show results from a two-dimensional transport calculation. The readjustment of fields and fluxes imply that three-dimensional motions and reconnection are returning the Spheromak to the preferred state. The ability to adjust itself in this fashion during the decay has led to suggestions that a Spheromak could be maintained in steady state, a very advantageous property from the controlled fusion viewpoint. A source of 
helicity could steadily replace the helicity lost due to resistive decay, and the relaxation mechanism would distribute the fluxes appropriately (in a strictly two-dimensional, axisymetric state, the Spheromak is doomed to a finite lifetime by Cowlings theorem [7]). Recent results from the LASL plasma gun experiment, illustrated in Figs. 14 and 15, show that this is indeed possible. The plasma gun is used as a long-pulse helicity source, in either of the two modes shown in Fig. 14a,b. The measured polnidal field in these two cases is shown in Fig. 15. The field is sustained for several times the resistive decay time and is limited only by the time scale of the power supply, which is a capacitor in this case.

Stability

The Spheromak, while in some sense a preferred MHD state, is not unconditionally stable. A particularly dangerous instability is the "tilt" mode [8], where the Spheromak tumbles about an axis at right angles to the symmetry axis if the plasma is not sufficiently oblate (shorter in the axial direction than in the radial direction) or if conducting walls are too distant. In an externally applied field, the Spheromak field can reconnect with applied field as it tilts, as shown schematically in Fig. 16. Measured field profiles [5] consistent with the illustrations in Fig. 16a-d are shown in Fig. 17 wher: $y$ is a coordinate along a diameter through the center of the device, $z$ is along the symmetry axis and $x$ is normal to $y$ and $z$. Fig. 18 shows the measured fields in the final state consistent with Fig. 16e, i.e., a Spheromak flipped $180^{\circ}$ with the external flux completely reconnected and passing through the center of the torus.

Fig. 19 shows coniours of radius $x B_{T}$ for a flux-core-produced Spheromak undergoing a tilt instability. In this case, the Spheromak tilts about $90^{\circ}$ before reconnection completely destroys the configuration. 


$$
-8-
$$

\section{Conclusion}

Reconnection plays an essential role in all phases of existence for tine Spheromak. It is present during the formation and slow decay, as well as the rapid destruction of the Spheromak when an instability occurs. Reconnection in an intrinsically three-dimensional fashion raises the intriguing possibility that a Spheronak may be sustained indefinitely against resistive losses by the injection of magnetic helicity.

\section{Acknowledgments}

I would like to acknowledge helpful discussions with C. W. Hartman, T. R. Jarboe, J. B. Taylor, and W. C. Turner. The data presented in this paper were provided by G. C. Goldenbaum, M. Yamada, A. Janos, W. C. Turner, A. R. Sherwood, T. R. Jarboe, and C.W. Barnes. This work was performed under the auspices of the U.S. Department of Energy by the Lawrence Livermore National Laboratory under contract number W-7405-ENG-48. 
Some confusion exists regarding gauge invariance properties of the helicity as well as the appropriate generalization when magnetic field lines intercept the conducting boundaries. To clarify these issues consider the time derivative of the usual definition of the helicity

$$
\dot{K}=\left(\int \underline{A} \cdot \underline{B} d V\right)=\int \underline{A} \cdot \underline{B} d V+\int \underline{A} \cdot \underline{B} d V .
$$

The volume under consideration is assumed to be enclosed by conducting surfaces, possibly partitioned by infinitessimally thin insulators te allow for injection of magnetic flux. Field lines are allowed to intercept the walls. For the time being we restrict ourselves to gauges satisfying $\Phi \underline{A} \cdot \underline{d l}=$ enclosed magnetic flux. This restriction implies

$$
\underline{E}=-\underline{A}-\nabla \phi \quad
$$

where $\phi$ is a single valued function of space. Using Faraday's Law, $\underline{B}=-\nabla \times \underline{E}$, Gauss's Law, $\nabla \cdot \underline{B}=0$, and integrating by part gives

$$
\dot{K}=-2 \int \underline{E} \cdot \underline{B} d V-\int_{\text {surface }} \phi \underline{B} d s+\int_{\text {surface }} \underline{A} \times \underline{E} \cdot \underline{d s}
$$

If the volume is filled with conducting plasma, then $\underline{E} \cdot \underline{B} \cong 0$ (except in the neighborhood of reconnection regions, which presumab? y constitute a small portion of the volume at large magnetic Reynold's numbers) and the first term can be neglected. If no field lines penetrate surfaces $\underline{B} \cdot \underline{d s}=0$ and i the second t.erm vanishes. If no insulators are present, the condition $\underline{E} \times \underline{\hat{n}}=0$ at conducting surfaces causes the third term to vanish and therefore $\dot{k}=0$, the usual form of the helicity is preserved. In the more general case, 
including insulators ard allowing for $\underline{B} \cdot \underline{d s} \neq 0$, consider the condition $\underline{E} \times \hat{n}=0$ on conductors:

$$
E \times \hat{n}=-(\underline{A}+\nabla \phi) \times \hat{n}=0 .
$$

Equation A4 implies that on the conducting surface

$$
\phi(t, \underline{x})=\phi\left(t, \underline{x}_{0}\right)-\int_{\underline{x}_{0}}^{\underline{x}} \underline{A} \cdot \underline{d l}
$$

where $\underline{x}_{0}$ is an arbitrary point on the conducting surface. The function $\Phi$ can be different on each conducting surface separated by an insulator. Using Eq. (A5) in Eq. (A3) (neglecting the E - B terrr again by assuming the volume is filled with conducting plasma)

$$
\begin{gathered}
\dot{K}=\int_{\text {surfface }} \int_{\underline{x}_{0}}^{\underline{x}} \underline{A} \cdot \underline{d l} \underline{B} \cdot \underline{d s}-\sum_{i} \Phi_{i}\left(t, \underline{x}_{0}\right) \int_{\text {surfaces }} \underline{B} \cdot \underline{d s} \\
+\int_{\text {surface }} \underline{A} \times \underline{E} \cdot \underline{d s}
\end{gathered}
$$

the sum on $i$ corresponds to summing over the number of distinct surfaces separated by insulating gaps.

Since the elements of flux intercepting the conductors, $\underline{B} \cdot \underline{\text { ds }}$ are constant in time, the first term in Eq. (A6) can be brought over to the lefthand side:

$$
\dot{K}^{\prime}=-\sum_{i} \Phi_{i} \int \underline{B} \cdot \underline{d s}+\int \underline{A} \times \underline{E} \cdot \underline{d s}
$$


where

$$
K^{\prime}=\int \underline{A} \cdot \underline{B} d V-\int_{S L r f a c e}\left(\int_{\underline{x}_{0}}^{\underline{x}} \underline{A} \cdot \underline{d]}\right) \underline{B} \cdot \underline{d s}
$$

Note that $K^{\prime}$ is independent of the choice of $\underline{x}_{0}$ :

$$
K^{\prime}\left(\underline{x}_{1}\right)=K^{\prime}\left(\underline{x}_{0}\right)+\int_{x_{0}}^{\underline{x}_{1}} \underline{A} \cdot \underline{d l} \int_{\text {surface }} \underline{B} \cdot \underline{d s},
$$

However, since the volume is totally enclosed, $S \underline{B} \cdot \underline{d s}=0$ for the entire surface and $K^{\prime}\left(\underline{x}_{1}\right)=K^{\prime}\left(\underline{x}_{0}\right) . K^{\prime}$ is also gauge invariant, even if we drop the constraint that $\$ \underline{A} \cdot \underline{d l}=$ enclosed flux:

$$
\begin{aligned}
K^{\prime}\left(\underline{A}+\nabla_{\phi}\right) & =\int \underline{A} \cdot \underline{B} d V+\underline{\nabla \phi} \cdot \underline{B} d V \\
& -\iint \underline{x} A \cdot \underline{d l} \underline{B} \cdot \underline{d s}-\int \phi \underline{B} \cdot \underline{d s} \\
& =K^{\prime}(\underline{A})+\int_{\text {surface }} \phi \underline{B} \cdot \underline{d s}-\int_{\text {surface }} \phi \underline{B} \cdot \underline{d s} \\
& =K^{\prime}(\underline{A})
\end{aligned}
$$

where the surface integrals include any surfaces where $\phi$ may be multivalued. Next consider the right-hand side of Eq. (A7). The term AxE giv 35 a contribution only in the insulating gaps. For simplicity, consider for the moment only a single gap:

$$
\begin{aligned}
\int \underline{A} \times \underline{E} \cdot \underline{d s} & =\oint \underline{A} \cdot \underline{d l}\left(-\int \underline{E} \cdot \underline{d x}\right) \\
& \equiv V \psi
\end{aligned}
$$

where $V$ is the voltage across the gap, and $\psi$ is the flux linked by the gap. 
The other term in Eq. (A7) is given by

$$
\sum \Phi_{i} \int \underline{B} \cdot \underline{d s}=\Phi_{1} \psi_{1}+\Phi_{2} \psi_{2}
$$

$\Psi_{1,2}$ are the fluxes through the two distinct conducting regions. Flux conservation gives $\psi_{2}=-\psi_{1}=-\psi$, so the term becomes

$$
\sum \Phi_{i} \int \underline{B} \cdot \underline{d s}=\psi\left(\Phi_{1}-\Phi_{2}\right)
$$

From Eq. (A5) $\Phi_{1}-\Phi_{2}=\phi_{1}-\phi_{2}=-\int_{\text {gap }} \underline{E} \cdot d \underline{d x}=V$ so

$$
\sum \Phi_{i} \int \underline{B} \cdot d \underline{s}=\psi V
$$

and we have

$$
\dot{K}^{\prime}=2 \psi V
$$

For many gaps, the right-hand side of (A15) becomes simply $2 \Sigma_{i} \psi_{j} V_{j}=$ sum over all gaps of the flux linking the gap times the voltage drop across the gap. We then have

$$
K^{\prime}(t)-K^{\prime}(0)=2 \sum \psi_{i} \int v_{i} d t
$$

The integral $s V d t$ is the amount of flux injected through the gap which links the flux $\psi$, analogous to the simple case of two linked flux tubes where the helicity is twice the product of the linked fluxes. The generalized helicity $K^{\prime}$ given in Eq. $(A B)$ is therefore seen to be the correct extension to the case when flux penetrates coriducting walls, and to arbitrary gauges, as first suggested by J. B. Taylor [9].

The extra term, $j(s \underline{\mathrm{X}} \underline{A} \cdot \underline{d l}) \underline{B} \cdot \underline{d s}$, appearing in Eq. (AB) has a simple 
interpretation in the cases where $\int A \cdot \underline{d l}=$ enclosed flux. The integral could be rewritten as a sum over flux bundles penetrating the wall, $\mathrm{d} \psi=\underline{\mathrm{B}} \cdot \underline{\mathrm{ds}}$

$$
\int\left(\int \underline{\underline{x}} \underline{A} \cdot \underline{d l}\right) \underline{B} \cdot \underline{d s}=\int d \psi \int_{\underline{\alpha}}^{\underline{B}} \underline{A} \cdot \underline{d l}
$$

where $\underline{\alpha}, \underline{B}$ are the locations where the flux bundle $d \psi$ enters and exits the surface. Now consider a generalized system that includes the space exterior

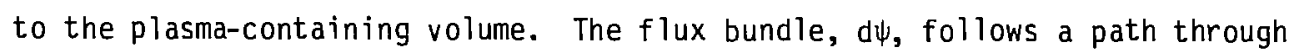
the exterior space from $\underline{B}$ to $\underline{\alpha}$, and we can write

$$
\int_{\text {surface }}^{\underline{B}} \underline{A} \cdot \underline{d l}+\int_{\text {exterior }}^{\underline{\alpha}} \underline{A} \cdot \underline{d l}=\oint_{\text {exterior }} \underline{A} \cdot \underline{d l}
$$

The right-hand side of $\mathrm{Eq} .(\mathrm{Al})$ is a constant, assuming the exterior space is unchanging in time so we can write

$$
\begin{aligned}
K^{\prime} & =\int_{\text {plasma }} \underline{A} \cdot \underline{B} d V-\int d \psi \int_{\underline{\alpha}}^{\underline{B}} \underline{A} \cdot \underline{d l} \\
& =\int_{\text {plasma }} \underline{A} \cdot \underline{B} d V+\int d \psi \int_{\underline{B}}^{\underline{Q}} \underline{\text { exterior }} \underline{A} \cdot \underline{d l}+a \text { constant } \\
= & \int_{A} \cdot \underline{B} d V \mid \text { extended volume }+ \text { a constant }
\end{aligned}
$$

The generalized helicity, $K^{\prime}$, is therefore equivalent to extending the volume of integration to include the closure of all flux loops penetrating the conducting boundaries. This is often a useful way to calculate $K^{\prime}$ in complex geometries. Since $K^{\prime}$ is a topological property of the fields, the exterior region can be idealized as long as the linkages with the fields in the plasma volume are preserved. 
References

[1] M. Yamada, et. al., Phys. Rev. Lett. 46, 188 (1981).

[2] G. C. Goldenbaum, J. H. Irby, Y. P. Chong, and G. W. Hart, Phys. Rev. Lett. 44, 393, (1980).

[3] H. Alfven, L. Lindberg, and P. Mitlid, J. Nucl. Energy Part C, 116 $(1960)$.

[4] T. R. Jarboe, I. Henins, A. R. Sherwood, Chris W. Barnes, and H. W. Hoida, Phys. Rev. Lett. 51, 39 (1983).

[5] W. C. Turner, et. al., Phys. Fluids 26, 1965 (1983).

[6] J. B. Taylor, Phys. Rev. Lett. 33, 1139 (1974).

[7] T. G. Cowling, Mon. Notic. Roy. Astron. Soc. 94, 39 (1934).

[8] M. N. Rosenbluth and M. N. Bussac, Nuc1. Fus. 19, 489 (1979).

[9] J. B. Taylor, private communication. 
Figure Captions

Fig. 1 Inductive Spheromak formation using a flux core. Poloidal magnetic field surrounding the toroidal core is pre-established. Toroidal field is induced into a plasina by the currents flowing in the core and the plasma pinches radially inward, reconnecting and forming a Spheromak .

Fig. 2 Z-theta pinch Spheromak formation. The symmetry axis is at right angles to the axis in Fig. (1). After pre-establishing a bias field in the $z$ direction, a current is drawn in the $z$ direction by electrodes, producing a toroidal field. The azimuthal, single turn coil is then energized, producing a field that opposes the bias field. Reconnection and relaxation to a Spheromak equilibrium follows.

Fig. 3 Coaxial plasma gun formation of a Spheromak. Poloidal flux threading the coaxial gun is pre-established. A current is drawn across the gun electrodes, producing a toroidal field that stretches the poloidal lines through an entrance region and into a flux conserving chamber. The field in the entrance region reconnects, leaving an isolated Spheromak .

Fig. 4 Poloidal flux map from a $z-\theta$ pinch formation experiment.

Fig. 5 Contours of radius $\times \mathrm{B}_{\mathrm{T}}$ for different times during Spheromak formation in a flux core experiment.

Fig. 6 The upper figure shows decay of the field in the entrance region of a plasma gun experiment. The lower figure shows the slower decay of the Spheromak field. All times are in microseconds.

Fig. 7 Axial field in the entrance region of a plasma gun experiment: (a) shows the field due to a pinching coir alone; (b) shows the field due to the plasma gun alone; (c) shows the combined fields. 
Fig. 8 (a) A flux tube with a half twist above a reference plane; (b) the integration path for evaluating $\Phi \underline{A} \cdot \underline{d l}$; (c) the integration path split into two pieces; the first encloses no flux and the second encloses the flux tube.

Fig. 9 (a-d) Transformation of a simple knot into three twists by reconnection. Fig. 10 Flux amplification in plasma gun formation of a Spheromak.

Fig. 11 Comparison of experimental field profiles (boxes) to theoretical, force free profiles (solid curves) in a plasma gun experiment.

Fig. 12 observed poloidal flux in the Spheromak produced by a plasma gun as a function of (a) volt seconds introduced through the gun insulator, and (b) poloidal flux in the plasma gun. Solid curves are theoretical predictions.

Fig. 13 Decay of fields in a plasma-gun-produced Spheromak.

Fig. 14 Schematic field line patterns in a plasma-gun-sustained Spheromak. In (a) the Spheromak has the same orientation as the plasma gun fields. In (b) the Spheromak is rotated $180^{\circ}$ about an axis coming out of the page.

Fig. 15 observed poloidal field vs. time for the (a) normal, and (b) flip sustained modes illustrated in Fig. 14.

Fig. 16 (a-e) Field line patterns during tilting and reconnection of a Spheromak.

Fig. 17 (a-d) Observed field profiles in a plasma gun experiment consistent with Fig. $16(a-d)$.

Fig. 18 observed field profiles in a plasma gun experiment consistent with Fig. $16(\mathrm{e})$.

Fig. 19 Contours of radius $\times B_{T}$ for a Spheromak undergoing a tilt instability in a flux core experiment. 

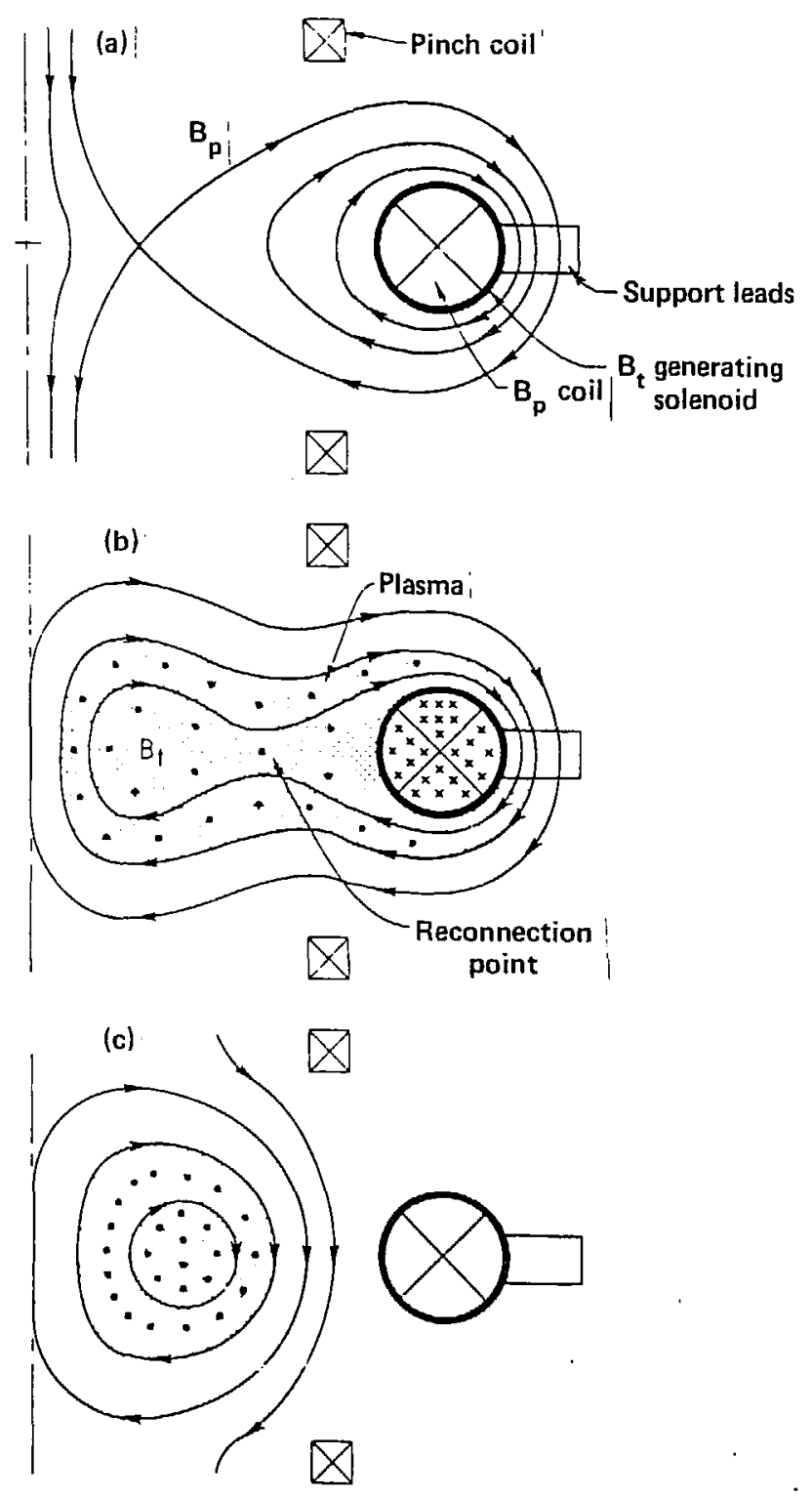

Fig. 1 

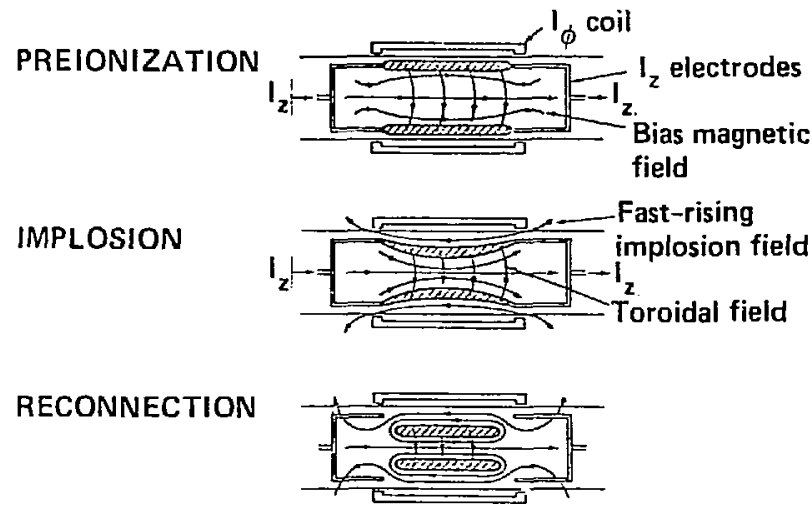

EOUILIBRIUM

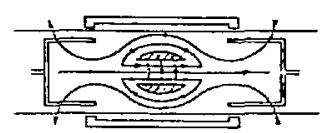

Fig. 2 

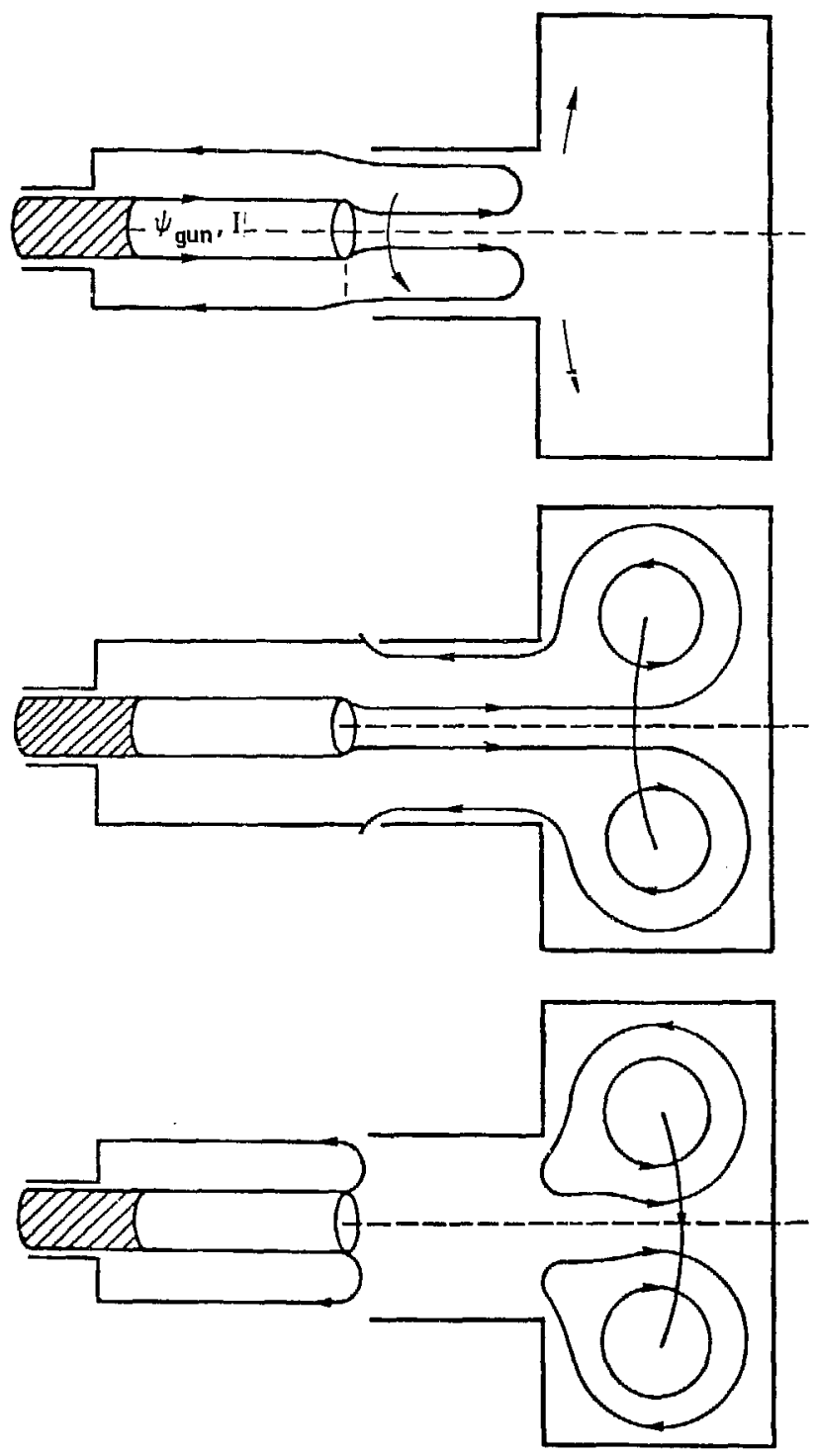

Fig. 3 


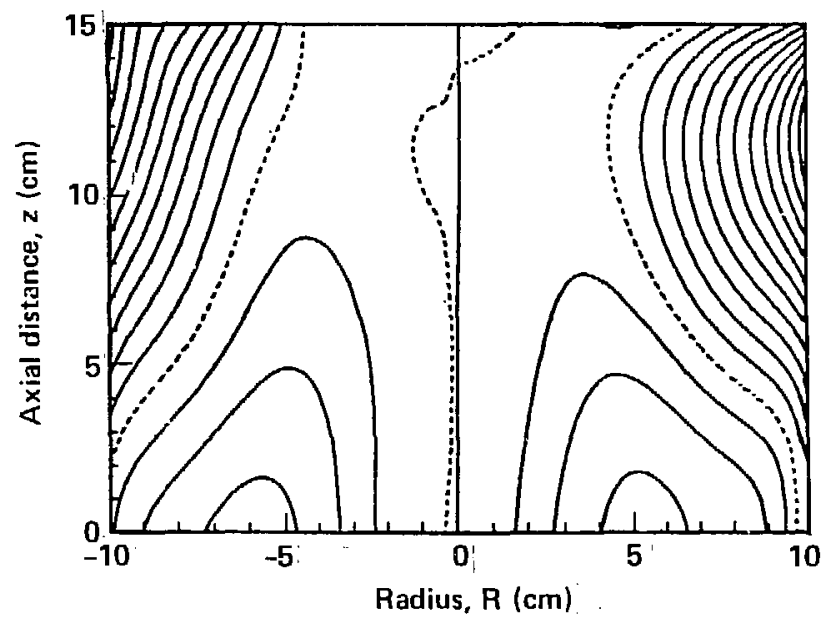

Fig. 4 


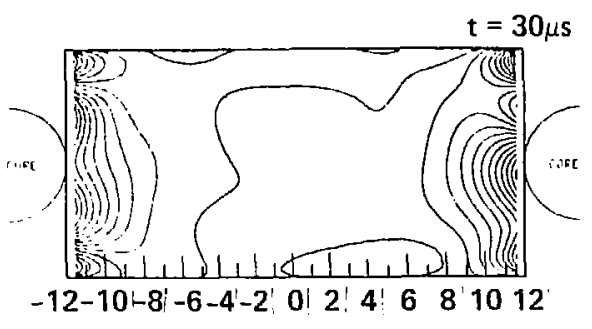

Contour plot of $B_{t}$ on the $r-z$ plane'
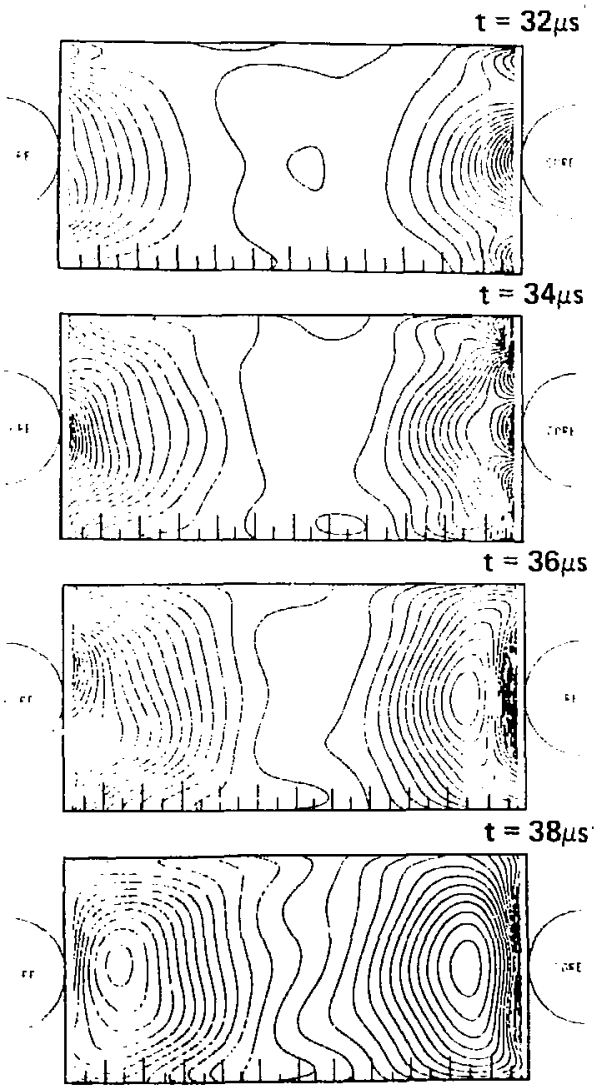
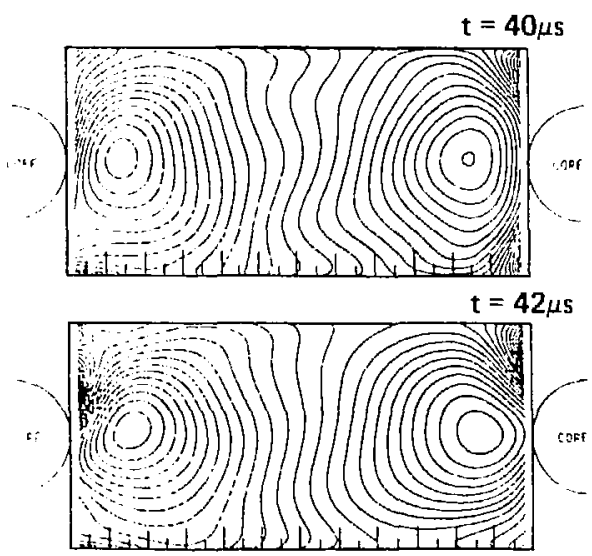

$\mathrm{t}=44 \mu \mathrm{s}$
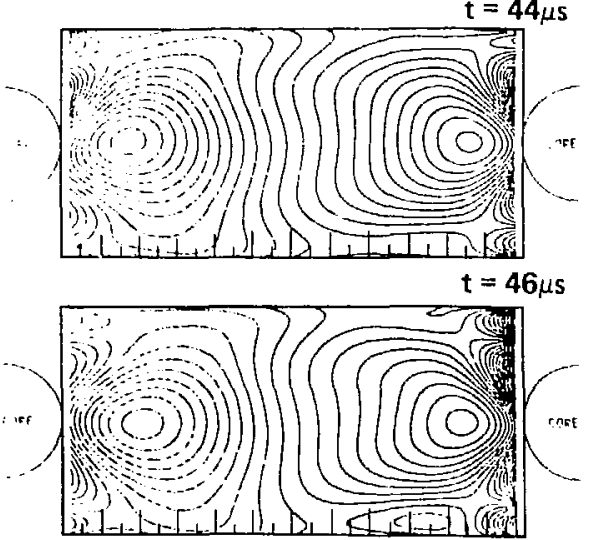

Fig. 5 


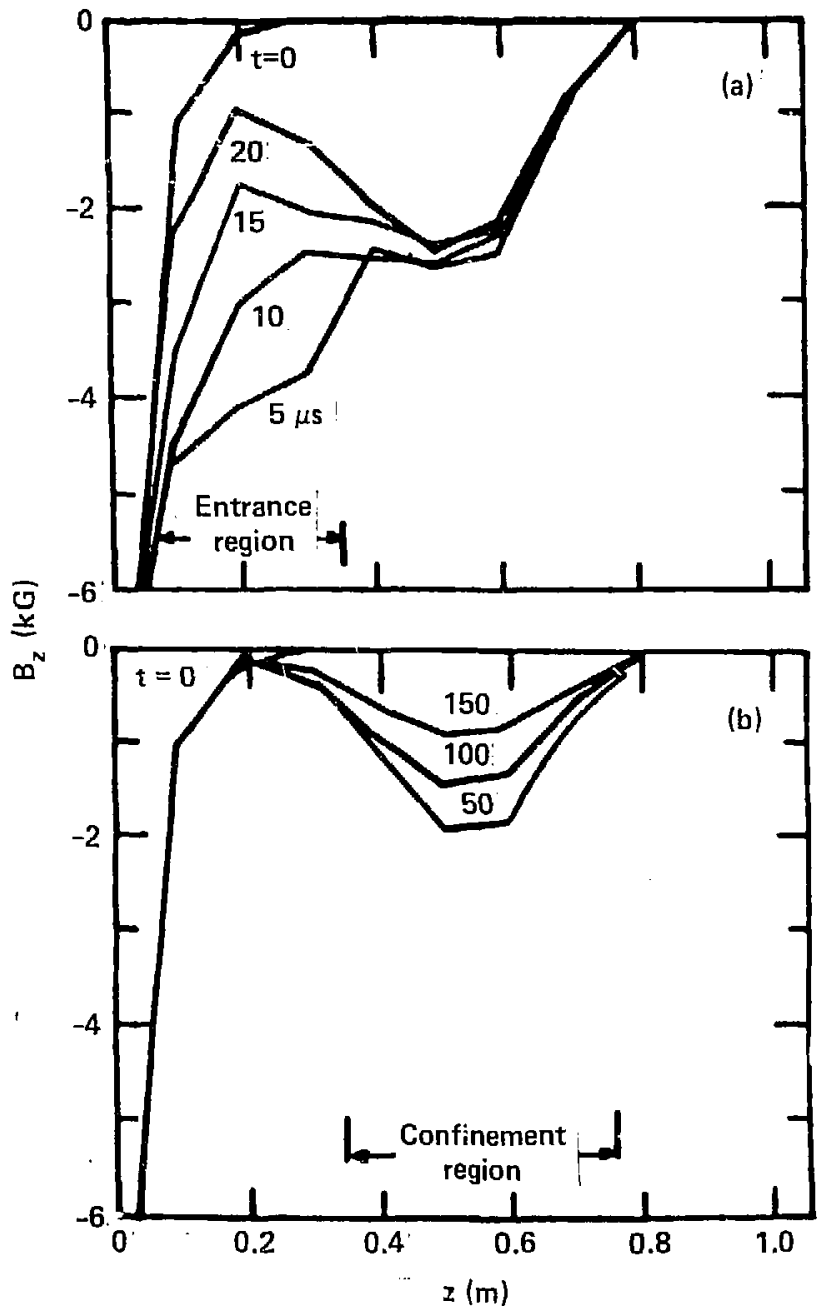

Fig. 6 

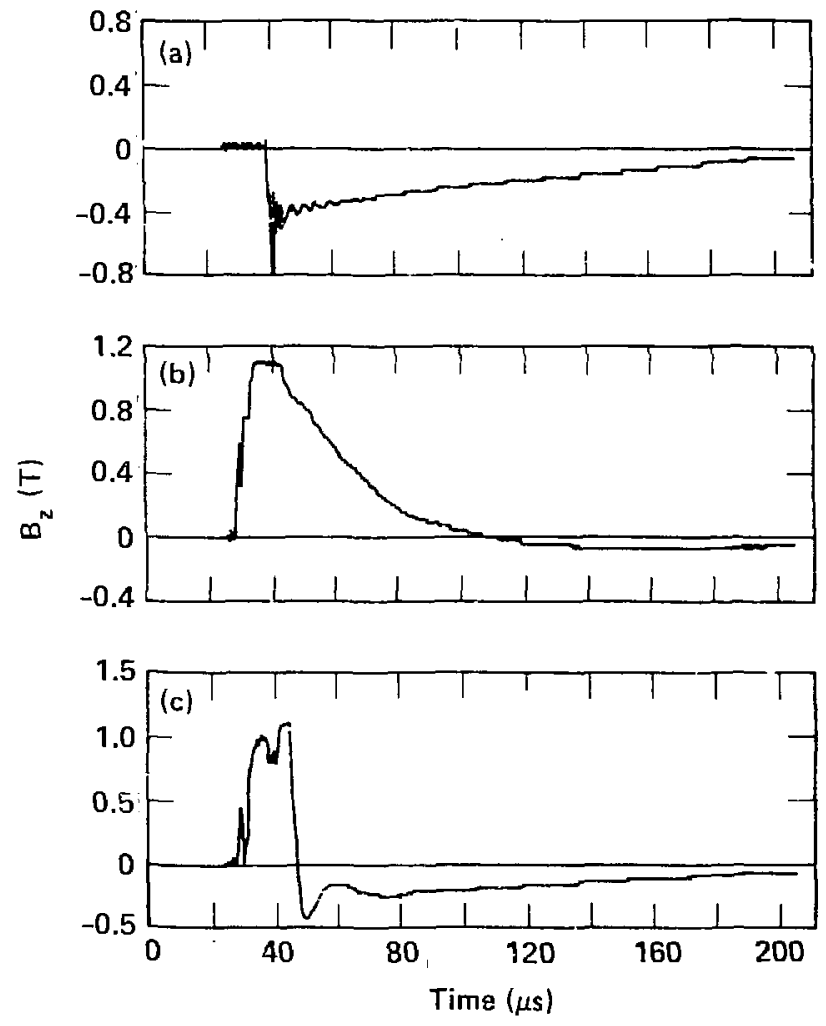

Fig. 7 
$-24-$

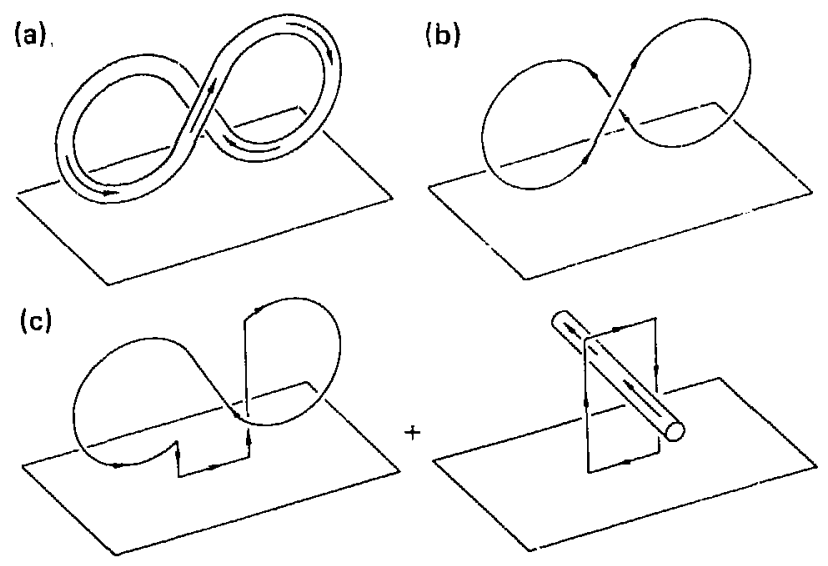

Fig. 8 
(a)

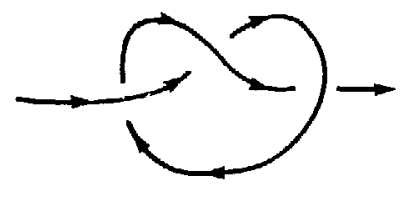

(c)

000 (b)

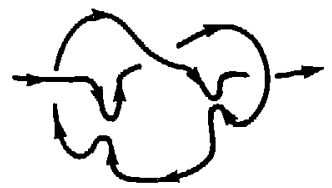

(d)

000

Fig. 9 


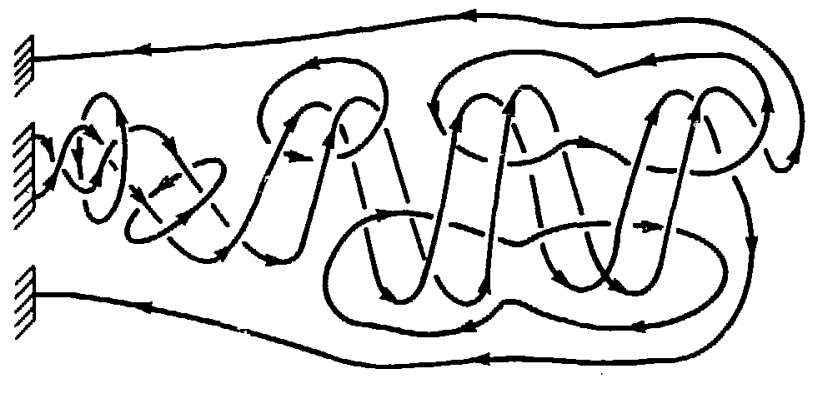

Fing. 10 


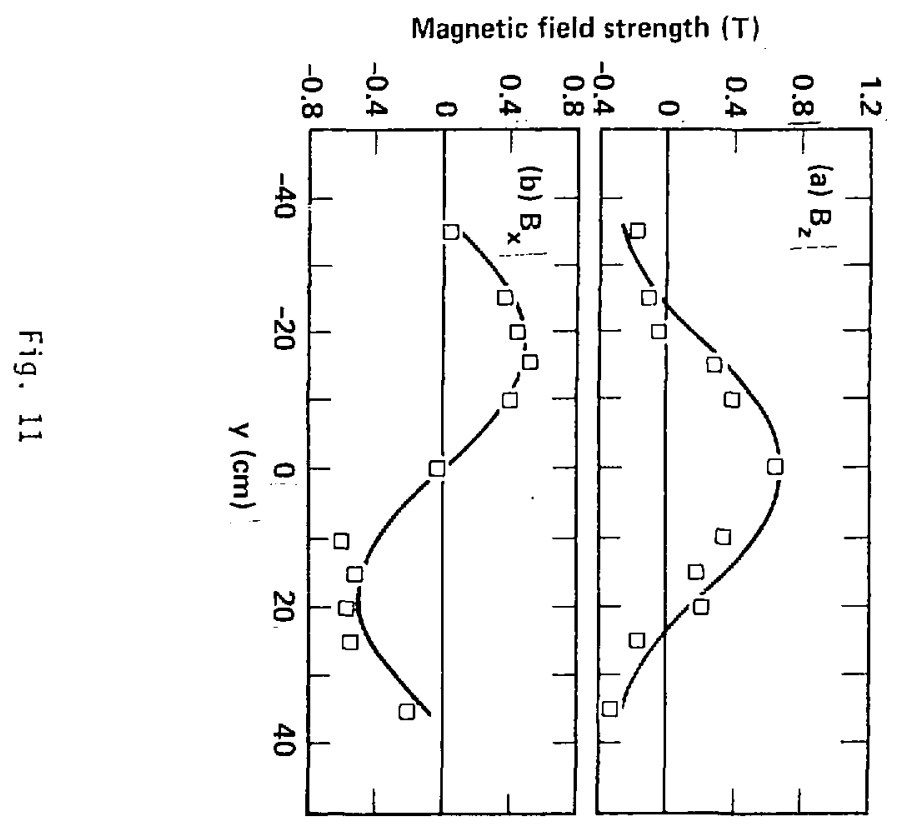




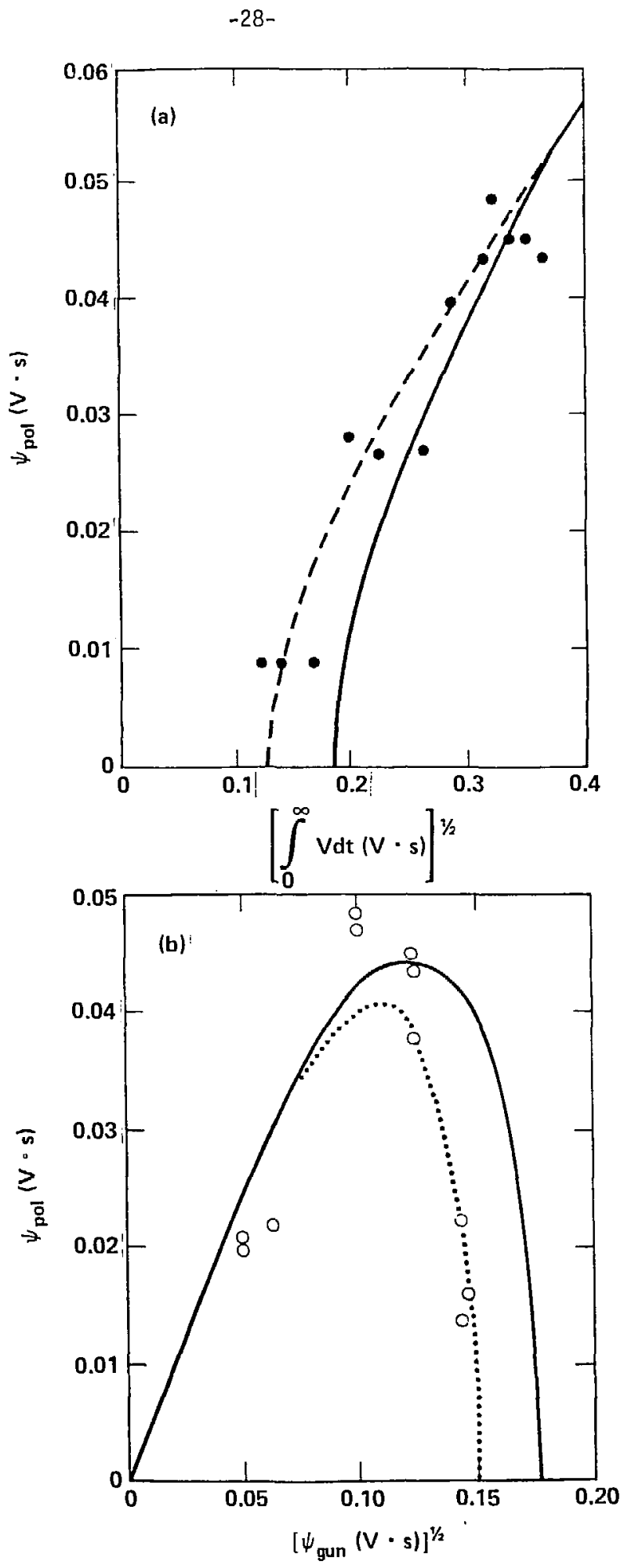

Fig. 12 

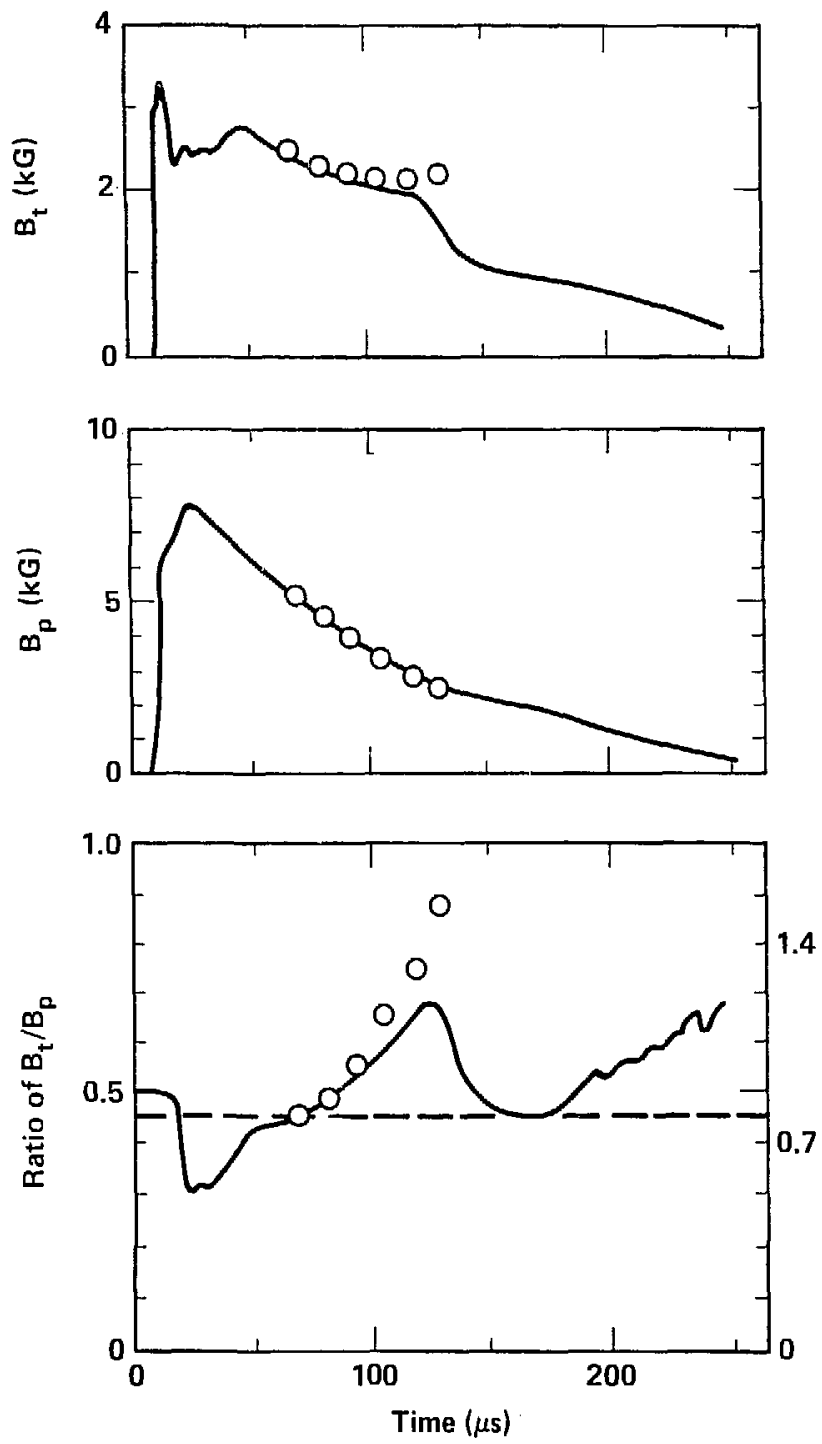

Fig. 13 

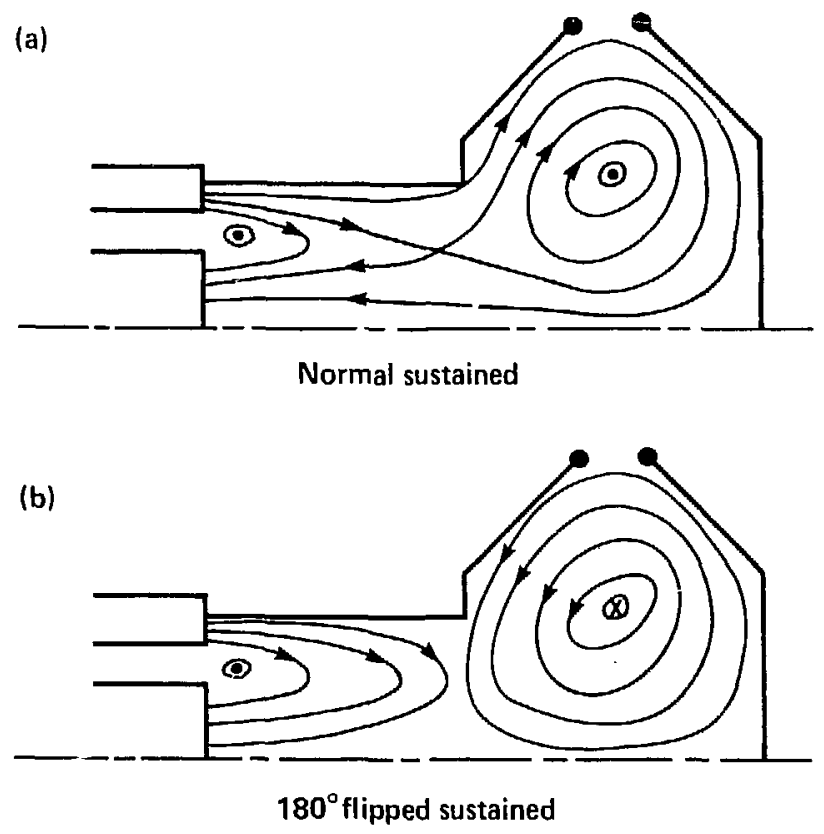

Fig. 14 


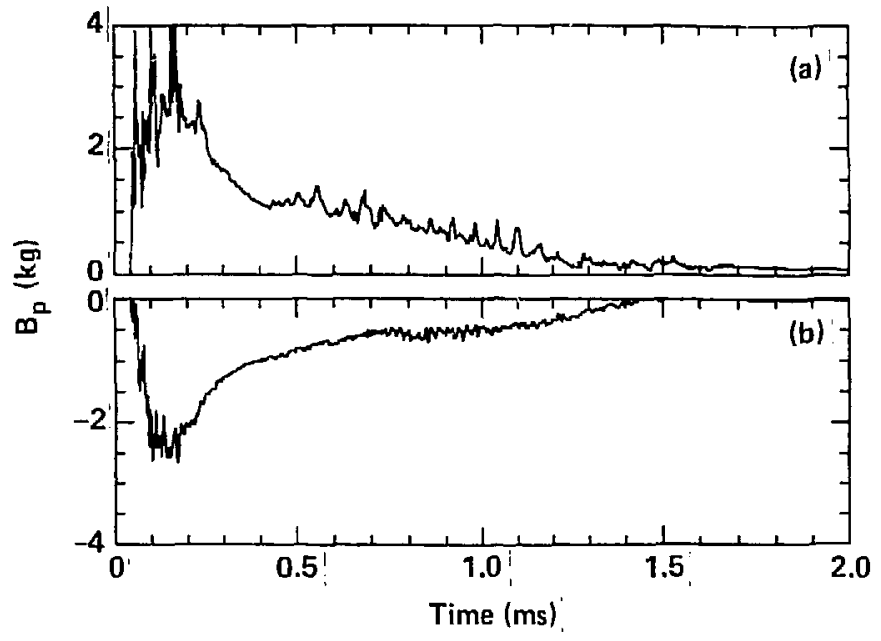

Fig. 15 

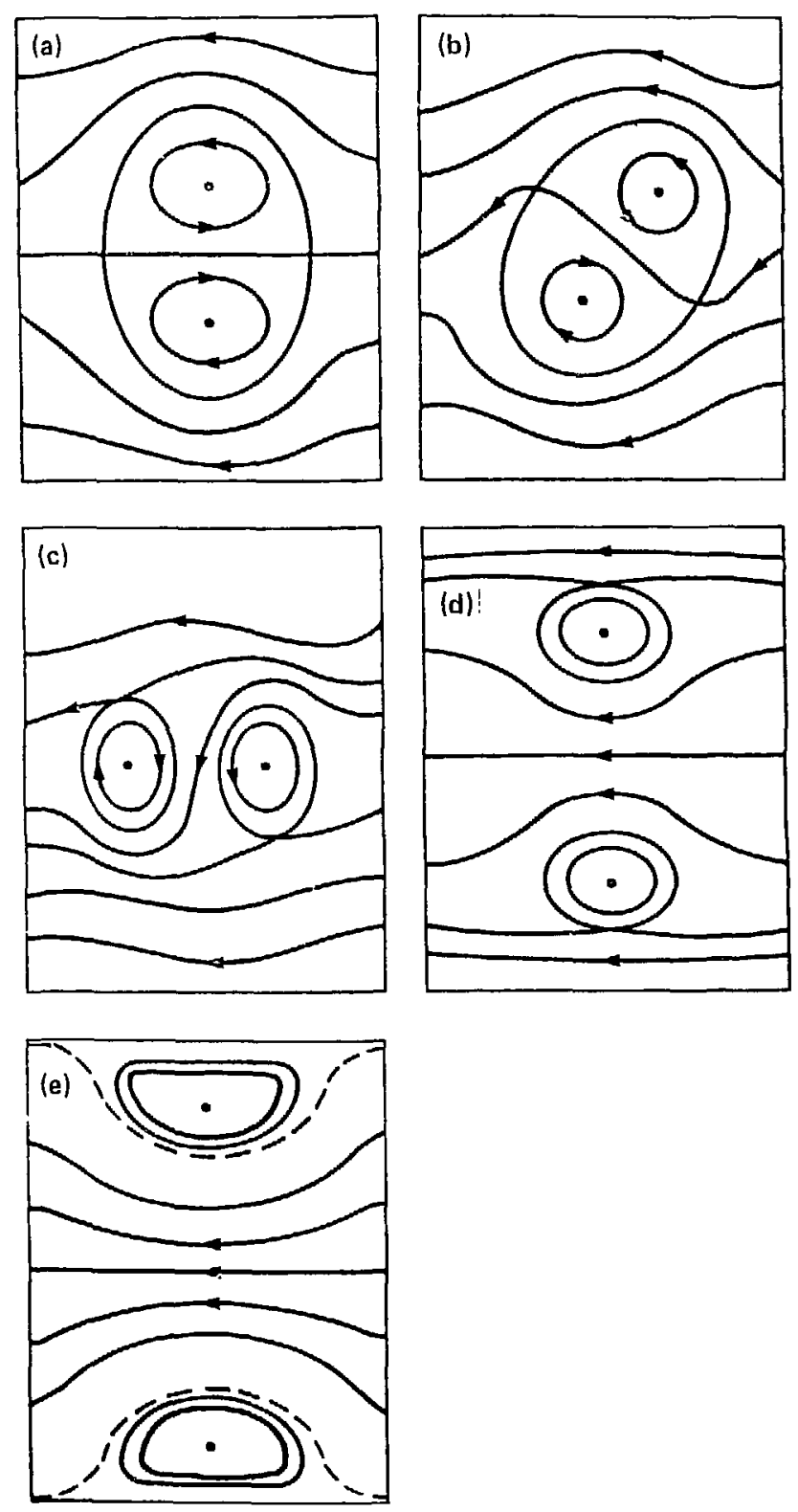

Fig. 16 

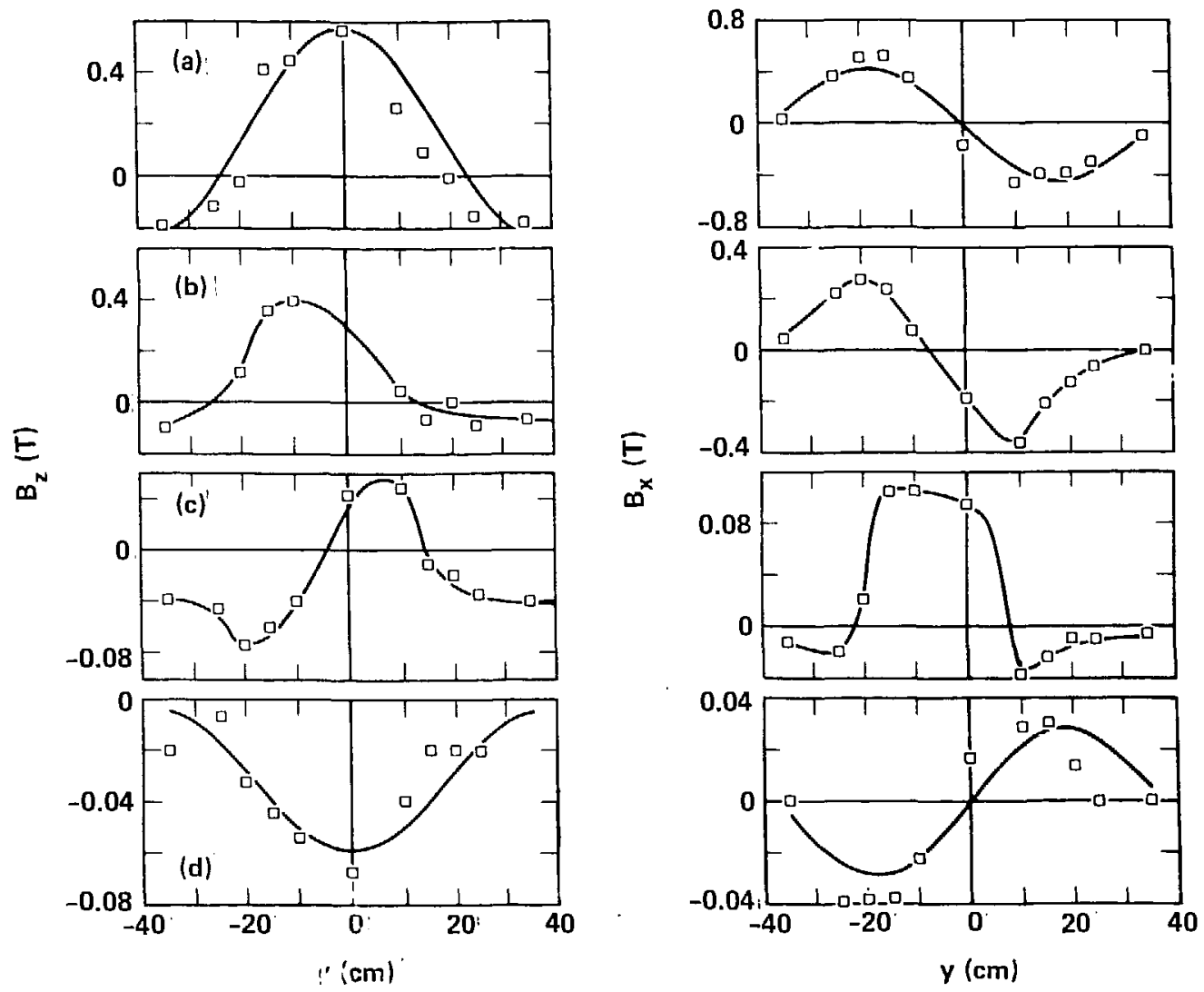

Fig. 17 

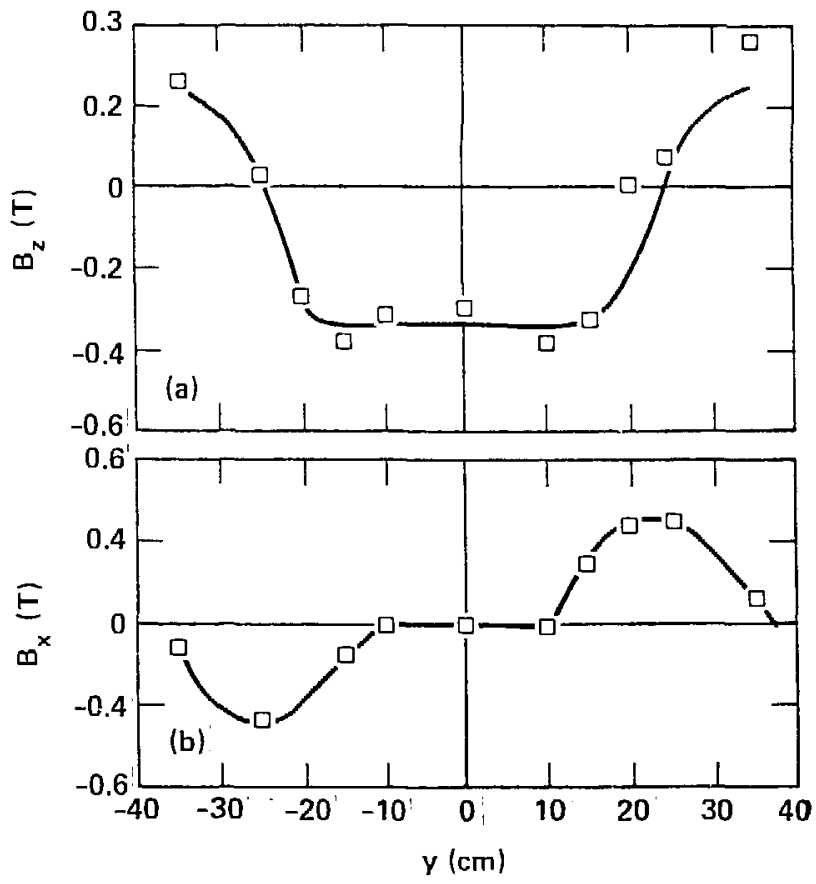

Fig. 18 

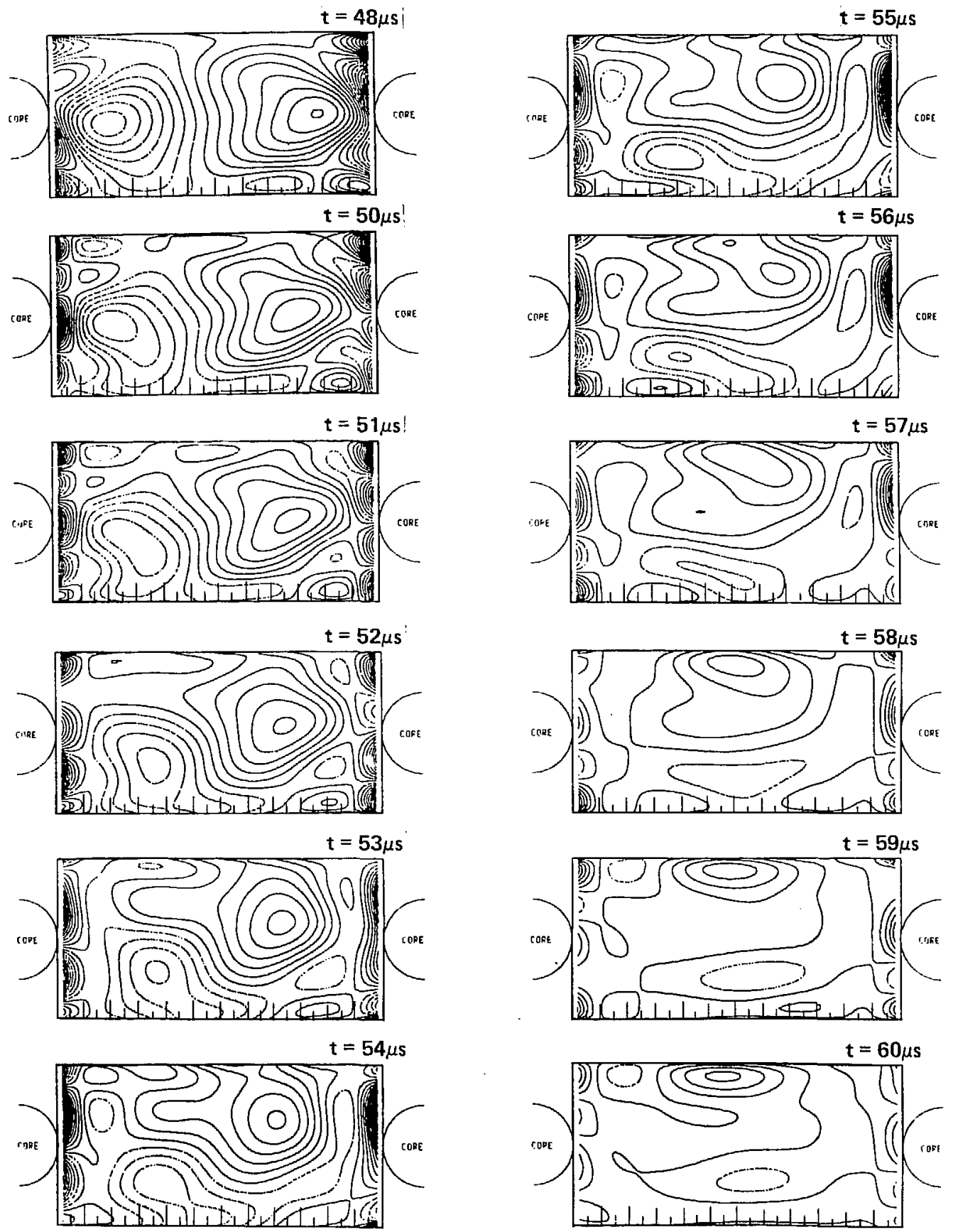

Fig. 19 